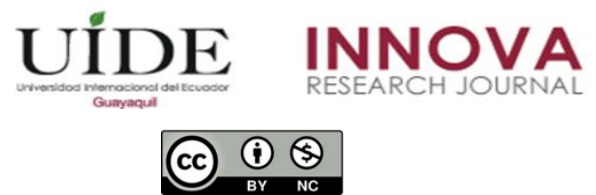

INNOVA Research Journal, ISSN 2477-9024

(Mayo-Agosto 2021). Vol. 6, No.2 pp. 180-195

DOI: https://doi.org/10.33890/innova.v6.n2.2021.1636

URL: http://revistas.uide.edu.ec/index.php/innova/index

Correo: innova@uide.edu.ec

\title{
Estrategias post COVID-19: Desafíos para las empresas exportadoras de productos tradicionales
}

\section{Post COVID-19 strategies: Challenges for companies exporting traditional products}

Nerissa Mischell Ulloa-Barre

Universidad Técnica de Machala, Ecuador

nmulloa_est@utmachala.ecu.ec

(D) https://orcid.org/0000-0002-5099-8628

Viviana Alexandra Fajardo-Loayza

Universidad Técnica de Machala, Ecuador

vafajardo_est@utmachala.edu.ec

(i) https://orcid.org/0000-0001-8347-4495

Mayiya Lisbeth González-Illescas

Universidad Técnica de Machala, Ecuador

mlgonzalez@utmachala.edu.ec

iD http://orcid.org/0000-0002-5219-3807

Sandra Sayonara Solórzano-Solórzano

Universidad Técnica de Machala, Ecuador

ssolorzano@utmachala.edu.ec

(D) http://orcid.org/0000-0001-6294-7396

Recepción: 26/11/2020 | Aceptación: 17/02/2021 | Publicación: 10/05/2021

Cómo citar (APA, séptima edición):

Ulloa-Barre, N. M., Fajardo-Loayza, V.A., González-Illescas, M. L., y Solórzano-Solórzano, S. S. (2021). Estrategias post COVID-19: Desafíos para las empresas exportadoras de productos tradicionales. Innova Research Journal, 6(2), 180-195.

https://doi.org/10.33890/innova.v6.n2.2021.1636

\section{Resumen}

En el contexto del comercio internacional, la crisis sanitaria desatada por el COVID-19, ha influenciado en las actividades laborales y en el ambiente de negocios. La capacidad de adaptación de las empresas exportadoras cobra mayor importancia para defender su permanencia en el 
mercado. Este trabajo tiene por objetivo identificar estrategias que permitan a las empresas exportadoras adaptarse a las transformaciones derivadas del COVID-19. Se empleó un enfoque cualitativo, con la aplicación de los métodos teóricos analítico-sintético para analizar y caracterizar las estrategias empresariales y los desafíos comerciales. Los resultados obtenidos señalan que la diversificación de mercados, de proveedores, la digitalización de operaciones y el comercio electrónico han sido las estrategias más recomendadas para enfrentar la crisis sanitaria. Se concluye que, si bien estas estrategias no cubren todas las necesidades de las empresas exportadoras, pueden servir de referente para robustecer el aprendizaje en un escenario inesperado que ha afectado de forma desigual a las empresas alrededor del mundo.

Palabras claves: Exportaciones post Covid-19; impacto COVID-19; estrategias de exportación.

\begin{abstract}
In the context of international trade, the global health crisis released by COVID-19 has influenced the way people used to work and the entire business environment. The adaptability of exporting companies becomes more important to be able to defend their permanence in the market. This work aims to identify strategies that allow exporting companies to adapt to the transformations consequent from COVID-19. Throughout the investigation, a qualitative approach was used, with the application of analytical-synthetic theoretical methods to analyze and characterize business strategies and business challenges. The results obtained indicate that the diversification of markets, providers, the digitization of operations and, more importantly, electronic commerce (ecommerce) have been the most recommended strategies to face the health crisis. From the present investigation, it is concluded that, although these strategies do not cover all of the needs of exporting companies, they can serve as framework to strengthen skills in an unexpected scenario that has unevenly affected companies around the world.
\end{abstract}

Keywords: Post COVID-19 Exports; Impact of COVID-19; Exporting Strategies.

\title{
Introducción
}

La pandemia desatada por el COVID-19 ha provocado afectaciones en la salud y en la vida de las personas. El temor de la sociedad por el contagio, ha conducido a distorsiones en los patrones de consumo (Baldwin y Weder di Mauro, 2020).

Las restricciones a la libre circulación, adoptadas por los gobernantes a comienzos de la crisis sanitaria, interrumpieron la producción y los flujos de comercialización a nivel mundial, haciendo visible la desaceleración económica (ALADI, 2020). La contracción de las actividades productivas en los mercados de Asia, Europa y Estados Unidos, ocasionó desempleo, reducción del poder adquisitivo y disminución en la demanda de bienes (Banco Mundial, 2020).

El interés por el análisis de los efectos del COVID-19 en las exportaciones se refleja en una serie de informes de organismos internacionales como el Banco Mundial (2020), Organización Mundial de Comercio (2020), la Comisión Económica para América Latina (CEPAL, 2020), Asociación Latinoamericana de Integración (ALADI, 2020), los cuales hacen mención a las medidas de control y prohibiciones que han seguido los países. Se destaca el impacto en el nivel de oferta y en los precios de productos de exportación. 
Con respecto al sector importador de alimentos originarios de América Latina, se evidencia una baja sensibilidad de la demanda a la contracción económica, lo que refiere sobre el tipo de bienes esenciales para la alimentación. Bajo este contexto, se han identificado retrasos en las aduanas, debido a los controles para disminuir el ritmo de propagación del virus, como cuarentena en las instalaciones portuarias, protocolos sanitarios, distanciamiento social obligatorio de los trabajadores, cierre de carreteras.

Para Ecuador, la exportación de productos agropecuarios se constituye como la principal fuente de ingresos (no petroleros), siendo China, Estados Unidos y Europa sus principales mercados. El nuevo escenario ha planteado a las empresas ecuatorianas la exigencia de adaptación. En este sentido, se explica la preocupación central de este trabajo, identificar el impacto de la crisis sanitaria en las exportaciones ecuatorianas. En este contexto de antecedentes, el objetivo principal de esta investigación es identificar estrategias que permitan a las empresas exportadoras adaptarse a las transformaciones del ambiente de negocios, derivadas del COVID-19.

Para dar respuesta a la interrogante planteada y el objetivo de investigación, se emplea un enfoque cualitativo, de tipo descriptivo, con la aplicación de los métodos teóricos: analítico sintético e inductivo deductivo. La recopilación de información se realiza de organismos oficiales relacionados al comercio exterior y artículos científicos que tratan de prácticas organizacionales sugeridas por diversos autores para defender la participación de las empresas exportadoras en los mercados.

La estructura del documento presenta la introducción, seguida de la revisión de literatura que contiene un análisis de la economía mundial en tiempos de pandemia, aspectos de la estrategia empresarial y los desafíos que representa el COVID-19 en el contexto del comercio internacional. Se expone la metodología que sustenta la forma de responder la pregunta y objetivo de investigación. Posteriormente, los resultados exponen la recopilación de información asociada a las exportaciones y las estrategias sugeridas por otros autores para enfrentar la crisis sanitaria. Finalmente, en el apartado de conclusiones se puntualizan aspectos asociados a los resultados y futuras líneas de investigación.

\section{Revisión de la literatura}

\section{Economía mundial en tiempos de pandemia}

Quevedo et al. (2020), asumen que "el mundo, en la actualidad atraviesa una pandemia cuyas consecuencias afectan no solo la salud humana, sino que ha permeado las esferas sociales y económicas de todas las naciones" (p. 1008). Uno de los efectos más visibles, la ralentización de los intercambios comerciales internacionales (Gruszczynski, 2020).

El inicio de la crisis sanitaria en Wuhan (China), desde el mes de diciembre del 2019, ha provocado afectaciones a nivel mundial. Este nuevo panorama de salud pública generó dos importantes fenómenos a nivel de la economía global, y consecuentemente en el comercio internacional: un shock de la oferta y un shock de la demanda (Gómez, 2020). Estos eventos se reflejan en la disminución de las transacciones comerciales, descenso de la producción, la reducción de la jornada laboral y de salario (Foladori y Delgado, 2020) creando un efecto dominó 
en la economía mundial (Sorroza et al., 2020). En línea con las repercusiones descritas, la diminución de la demanda del mercado influye en la capacidad de financiamiento de las empresas y las posibilidades de futuras inversiones.

En el ámbito de la economía latinoamericana, el shock de la oferta y la demanda redujo los ingresos que provienen de las exportaciones de materia prima, al igual que el menor acceso a los mercados internacionales debido a las políticas acogidas por cada gobierno, como medida de protección para su población y su producción nacional.

De acuerdo al análisis de organismos mundiales, la profundidad de la recesión en países ricos mantendrá deprimidas las exportaciones al menos por lo que resta del presente año; considerando que el efecto se prolongará al año venidero (Banco Mundial, 2020; CEPAL, 2020). He y Harris (2020) "Un pronóstico alternativo es que puede generarse un retorno lento, a causa del miedo prolongado en los consumidores que se preocupan por la salud y seguridad" (p. 178). De acuerdo con Nicola et al. (2020) se necesita una planificación a medio y largo plazo para reactivar la economía; idea que se complementa con la necesidad de fortalecer la cooperación internacional con la finalidad de mantener las cadenas de suministro en funcionamiento en tiempos de crisis (Kerr, 2020). Sin duda, los países buscarán financiamiento para sus economías recurriendo a organismos internacionales como el FMI o el Banco Mundial.

Una pandemia establece en algunos casos la paralización de la economía, debido a la globalización la fuerza laboral de una empresa se debilita, su producción disminuye, por consecuencia la participación de su producto en el mercado se reduce. Sorroza et al. (2020) "Si se extrapola esta situación a muchas empresas, la economía del país sufre un desabastecimiento, no se generan ingresos y se empieza a afectar la economía y sus exportaciones" (p. 19).

\section{La estrategia empresarial}

Los autores clásicos como March y Simon (1958), señalan que la estrategia está relacionada con el pensamiento racional de los seres humanos, quienes toman decisiones en función de la información disponible. Así, la formulación y ejecución de la estrategia corresponden a rutinas organizativas enmarcadas en la información que se obtiene, de manera que dicha racionalidad es limitada. Para Chandler (1962), la estructura organizacional y la estrategia condicionan la adaptación al entorno. Hannan y Freeman (1977), destacan que las fuerzas competitivas y situaciones que se generan en el entorno, se constituyen en pautas para el diseño de estrategias.

Recurriendo a autores contemporáneos, se destaca la idea de Slusarczyk y Morales (2016) quienes indican que la estrategia empresarial describe generalmente cómo se logran los objetivos utilizando los recursos que la empresa posee. Como los recursos disponibles son limitados, la estrategia toma más importancia e implica el establecimiento de metas, anticipación de la conducta de los demás (competidores), determinación de las acciones y la movilización de recursos para la ejecución de las acciones nombradas.

De acuerdo a Alaña et al. (2018), la estrategia se representa en una planificación que define las acciones para lograr un propósito comercial tales como la forma de competir, el diseño de una 
ventaja competitiva, las fuentes de generación de valor en los productos y los medios para cubrir la demanda de los productos.

Toda estrategia requiere una adaptación a los nuevos paradigmas, es decir, a cuestiones de crecimiento económico, que encarnan la responsabilidad comercial, lo que se presenta como uno de los mayores desafíos que las organizaciones deben confrontar en la actualidad (Pereira et al., 2019).

En este sentido, toda estrategia debe adaptarse a situaciones cambiantes, a tal punto que la capacidad de flexibilidad y adaptación de las empresas se constituye en un recurso organizacional clave. Finalmente, en consideración a la situación actual que se enfrenta, pueden surgir habilidades de aprendizaje en la toma de decisiones, las cuales aumentan las posibilidades de generar ventajas competitivas sostenibles en el tiempo.

\section{Desafíos comerciales post COVID-19}

Según Sagot (2020):

"La pérdida de dinamismo que ha experimentado la producción nacional, convertida en una contracción a raíz de la pandemia por el COVID-19, coloca a la economía ante un reto aún mayor para reactivar y poner en la ruta del crecimiento el aparato productivo nacional". ( $\mathrm{p}$. 22)

La etapa comercial actual es crucial, las empresas deben adaptarse o acoplarse a los desafíos, y es claro que existe un antes y después de las negociaciones comerciales. AmankwahAmoah (2020), las empresas están experimentando amenazas a su supervivencia y los desafíos muchas veces no son fáciles de superar. En el caso del fortalecimiento de la cadena de suministro, qué es todo un reto puesto a que algunos de los proveedores ya no están en condiciones de abastecer su actividad habitual, sabiendo esto, los encargados deben buscar nuevos proveedores y considerar sustituir sus materias primas.

Según Zelicovich et al. (2020):

"Con la OMC limitada en sus capacidades y presa de las tensiones que acompañan el reacomodamiento del orden internacional, los acuerdos regionales o preferenciales vuelven a tener un papel estratégico como modo de resolver las tensiones y fricciones del comercio internacional". (p. 47)

Según Martínez y Rubio (2020) en su artículo referente a los emprendedores durante el COVID-19, es necesario replantearse un nuevo diseño dentro de las empresas para hacer frente a la situación compleja e insegura. Para ello, se debe hacer uso de todos los recursos disponibles e identificar y explotar oportunidades de negocio.

Haciendo referencia a lo citado por los autores, en la actualidad las empresas confrontan realidades inciertas, tras advertencias a su supervivencia que cada día se hacen más intensas, debiendo considerar los requerimientos de sus clientes y en particular de los países con mayores 
restricciones. Por lo tanto, las empresas deben garantizar la inocuidad de sus productos de exportación.

Así mismo, adaptarse a la digitalización es uno de los retos de mayor importancia, ya que en tiempos de crisis la automatización para liderar tareas administrativas y operativas, sirve para brindar soporte a la organización (Saavedra, 2020).

Según la OMC el comercio se ajustará a las disposiciones impuestas para de esta manera sobrellevar este estado y dar una solución a la crisis, adoptando los acuerdos regionales y preferenciales como una salida a las restricciones impuestas por medio de los gobiernos como medida de protección.

\section{Metodología}

El presente artículo se desarrolla bajo una perspectiva cualitativa, enfocada en el análisis y caracterización de las estrategias empresariales y los desafíos comerciales. Se aplica el método teórico analítico-sintético, para interpretar las estrategias que han implementado las empresas exportadoras para afrontar los retos ante el COVID-19.

La investigación es de tipo descriptivo, mediante la cual se exponen datos estadísticos de las exportaciones ecuatorianas, además de estrategias y desafíos empresariales post COVID-19. Para la recopilación de información se utilizan fuentes secundarias, artículos científicos e información de páginas de organismos oficiales ecuatorianos y otros relacionados con el comercio internacional.

Las estrategias seleccionadas, son de gran importancia para recuperarse de la crisis que está ocasionando la pandemia. De acuerdo a la selección de las estrategias se eligió aquellas con mayor relevancia y viabilidad. En el subtema de exportaciones durante el COVID-19 (figura 1) se utilizó un esquema elaborado por los autores, de acuerdo a datos tomados del Banco Central del Ecuador. Para reflejar las estrategias (tabla 1) la información se recopiló de diferentes artículos nacionales e internacionales referentes al tema.

\section{Resultados}

En concordancia con la interrogante que guía esta investigación, y el objetivo planteado, los resultados se dividen en dos apartados claramente diferenciados.

En primer lugar, se describe la situación de las exportaciones de productos tradicionales, del primer semestre del año 2020. Posteriormente, y de acuerdo a la revisión de literatura realizada mediante investigación documental, se expone una recopilación de varias estrategias identificadas en el contexto del comercio internacional, derivadas de la necesidad de enfrentar la situación de la crisis sanitaria desatada por el COVID-19.

\section{Exportaciones de Ecuador en el primer semestre del 2020}


Los flujos del comercio internacional generan altos ingresos a partir de productos de valor agregado, no obstante, existen países cuyas economías dependen en gran parte de la exportación de productos en estado primario (León et al., 2016).

En Ecuador, los productos tradicionales han mantenido una mayor participación dentro de las exportaciones no petroleras, en paralelo a una mayor tasa de crecimiento anual (Verdugo y Andrade, 2018). Así también, los productos tradicionales se destacan en las extensiones de áreas de cultivo. El escenario comercial para Ecuador, en el primer semestre del 2020 ha estado atado a la recuperación económica y a la evolución del consumo de sus principales mercados: Asía, Europa y Estados Unidos.

A continuación, se realiza una comparación de la tasa de variación de las exportaciones de productos tradicionales, entre los meses de enero a julio del 2019 y el mismo período del 2020, tomando como referencia la fuente oficial del Banco Central del Ecuador (2020).

\section{Figura 1}

Tasa de variación por productos tradicionales caso ecuador

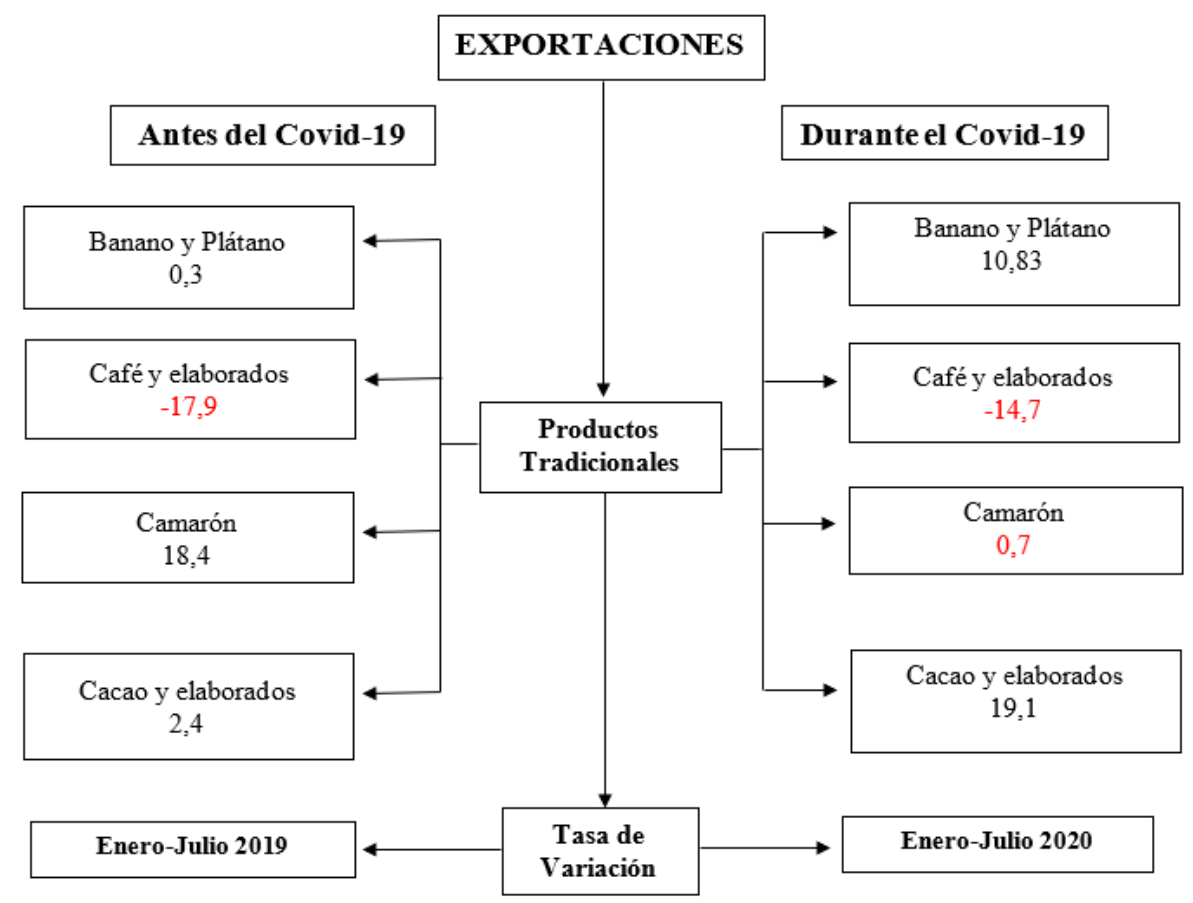

Elaborado por: Autoras

Fuente: Banco Central del Ecuador (2020)

En la comparación de los períodos expuestos, se destacan las exportaciones de banano y plátano, registrando un incremento del 10, 83\% en relación al año 2019. Otros países exportadores de banano como Costa Rica aumentaron sus ventas en 1,53\%, mientras que Colombia redujo su oferta exportable en 8,72\%. Según la Asociación de Exportadores de Banano del Ecuador (2020) los países como Alemania, Italia, Bélgica y Polonia (principales destinos del banano ecuatoriano en la UE) incrementaron sus compras en el primer trimestre del 2020 (p. 18). 
El ritmo de crecimiento de las exportaciones ecuatorianas de banano en junio de 2020 sufrió un retroceso en comparación a los meses anteriores. AEBE (2020) afirma que el total de volumen embarcado fue de 28'290.257 cajas, que, en comparación a mayo del 2020, significó una reducción de 8'071.751 cajas, equivalente a una disminución del 22\% en comparación al mes precedente (p. 19).

A pesar de las cifras del mes de junio, del primer semestre del 2020 se puede resaltar que las empresas exportadoras consiguieron mantenerse operativas, lo cual condujo a despachar la fruta a los mercados internacionales, sorteando aspectos logísticos relacionados con restricciones a la movilidad de personas y medios de transporte. Finalmente, las cifras del Banco Central del Ecuador (2020) indican que el banano se consolidó como primer producto de exportación no petrolero.

Con referencia al café y elaborados, se ve un notable descenso durante el primer semestre del año 2020 en comparación al año anterior del -17,9 al -14,7, Pablo Pinargote, gerente de Anecafé, indicó que en los cinco primeros meses del 2020 se logró exportar 164238 sacos, mientras que en el mismo lapso del año anterior fueron 166 976, es decir que la reducción fue del $2 \%$. (El Universo, 2020)

El producto que predomina en la exportación es el café industrializado, pese a las restricciones que se han dado las empresas dedicadas al negocio de café se han abastecido y han continuado con la comercialización del mismo, es por ello que el sector cafetero se ha mantenido.

En el sector cacaotero, los principales problemas que se presentaron fueron en el mes de marzo en cuanto a la comercialización y distribución del producto. Esto afecto a los pequeños productores que no podían efectuar el cobro. Los problemas logísticos fueron claros por las restricciones en cuanto al transporte que como sector exportador se debe efectuar para mantener el comercio internacional.

A diferencia del año 2019 se superó el tonelaje de exportación en los primeros 6 meses con un 18.974 TM. La actividad exportadora ha sido la clave de la economía del país durante los tiempos de COVID-19. Se tiene expectativas para el segundo semestre del año ya que en el mes de septiembre se da la cosecha más grande de cacao. (ANECACAO, 2020)

Respecto a las exportaciones de camarón, estas han mantenido un crecimiento constante, reforzado por las inversiones en las fincas camaroneras y las plantas de empaque que han mejorado la capacidad de producción. El crecimiento del año 2019 respecto al 2018 (período enero-julio) corrobora el impulso del sector camaronero. En el año 2020, si bien se observa un crecimiento del 0,7\%, las previsiones para el resto del año, según la Cámara Nacional de Acuacultura (Alvarado, 2020), no son halagadoras.

El mercado de China representó el 63\% de las exportaciones de camarón en el primer semestre del 2020 (Banco Central del Ecuador, 2020). Con el inicio de la pandemia en China, se paralizaron las importaciones de animales vivos, perjudicando el comercio de los países 
proveedores de camarón, langostas, salmón, etc., (Kakoolaki et al., 2020). Según Love et al., (2020):

"En algunos casos, entre los meses de enero a marzo, los distribuidores pudieron cambiar el comercio a otros mercados, como el camarón ecuatoriano congelado reencaminado de China a los Estados Unidos y Europa, luego de regreso a China en abril de $2020 ”$. (p. 4)

Es importante señalar que el camarón ecuatoriano en el mes de julio, enfrentó la suspensión de forma temporal de las importaciones de China. Concretamente, las autoridades aduaneras de China indicaron que los productos de tres empresas exportadoras de camarón dieron positivo a pruebas de COVID-19, asociándose a las paredes de los contenedores y empaques externos (Holguín, 2020).

En respuesta a esta situación, las empresas camaroneras fortalecieron sus sistemas de bioseguridad en la cadena productiva. Así, en Ecuador estableció a los exportadores de camarón, ciertas exigencias como medida sanitaria, como por ejemplo la emisión de un documento llamado Anexo Sanitario, en donde consta el nombre del producto a exportar, el tipo de producto, el resultado de los análisis, tipo de empaque, número de cajas, peso, y el número de referencia de los análisis. Este documento es adicional al certificado sanitario y se empezó a emitir a raíz de la pandemia para crear inocuidad en los procesos.

\section{Estrategias de exportación}

El COVID-19 ha generado grandes cambios en las actividades de comercio internacional, representando enormes desafíos para las empresas que se debaten entre la necesidad de mantener su productividad e innovar para hacer frente a las restricciones sanitarias y administrativas (Edeh, 2020), exigidas por los gobiernos para preservar la salud de su población.

Tras la disminución de la demanda a nivel internacional, las empresas exportadoras están incorporando estrategias para mantenerse vigentes en los mercados internacionales. En la tabla 1 se recopilan las ideas de algunos autores que han identificado formas de acoplarse a los nuevos requerimientos.

\section{Tabla 1}

Estrategias para enfrentar el covid-19

\section{AUTOR}

Baldwin y Evenett (2020)

\section{ESTRATEGIA}

La diversificación geográfica de la producción mejora la resiliencia de las cadenas de suministro al reducir la necesidad, y por tanto la probabilidad, de hacer frente a las restricciones y escasez de suministros. 
AUTOR

Barichello (2020)

Castro et al. (2020)
ESTRATEGIA

El gobierno de Canadá ha impuesto restricciones sanitarias y de seguridad a sus importaciones de alimentos. Siendo Canadá un país importador de productos de calidad, las certificaciones de producción orgánica y amigable con el medio ambiente, podrían ser la forma de atender a un grupo de consumidores que están dispuestos a pagar por la diferenciación. Esto puede incluir más inspecciones y valor agregado en el empaque minorista.

En el caso de las exportadoras de flores, las empresas deberán evaluar y reestructurar los procesos, productos, cadena logística y el transporte multimodal para mejorar los niveles de competitividad empresarial y su respectivo sostenimiento.

Oldekop et al. La digitalización en las actividades económicas ha sido una respuesta de las (2020) empresas frente a la necesidad de mantener el distanciamiento social. Así, el trabajo en línea y la logística organizada digitalmente han sido clave para mantener la operatividad. La adaptación a los canales digitales es una estrategia que ha permitido a las empresas enlazarse al comercio electrónico.

Fortalecer la cadena de suministro y los programas de financiamiento comercial para MIPYMES, Micro, pequeñas y medianas empresas (MIPYMES) que son

Park et al. (2020) particularmente vulnerables al impacto económico y comercial de COVID-19.
Las empresas exportadoras deben centrase en optimizar y diversificar las cadenas de suministro para hacer frente a los obstáculos dados por la crisis sanitaria. Es necesario diseñar una red de suministros, de tal manera que las empresas puedan equilibrar el riesgo y la flexibilidad operativa, gestionar las interrupciones de la cadena de suministro y mantener la cadena de suministro ágil.

\section{Subdirección General de Estudios y Evaluación de Instrumentos de Política Comercial (2020)}

España cuenta con una estrategia de internacionalización que se adapta a situaciones cambiantes como lo que sucede ahora con el tema del Covid-19, entre las estrategias ya en ejecución y que puede tomar aún más relevancia hoy se considera: el fomento a la diversificación de mercados de las exportaciones de bienes y servicios.

Fuente: Elaboración propia a partir de los autores citados.

Los resultados sugieren que, en situaciones no predecibles como la crisis sanitaria, es importante que los países exportadores puedan estar respaldados por planes alternativos para ofertar su producción. El fortalecimiento de las Tecnologías de la Información y Comunicación, ha contribuido en el desarrollo de un fuerte vínculo entre el comercio y la tecnología en general, permitiendo de esta manera acercarse a un mundo más digitalizado (Pesantez et al., 2020). 
Con ello también va de la mano, como ayuda a la oferta exportable, el apoyo del gobierno por medio de ferias internacionales virtuales para mantenerse en contacto con futuros clientes. Según Bonomelli (2020):

"Esta tendencia irá en aumento en los próximos años y nos invitará a acostumbrarnos a vivir y competir en un mundo del e-commerce, de ferias virtuales, de cambios en el marketing donde el trabajo incluya cada vez más a las TIC". (p. 11)

Otro de los hallazgos destaca que las empresas exportadoras, reconociendo afectación en su cadena de suministro, se han visto obligadas a conseguir nuevos proveedores, es decir, a la diversificación de los mismos. Así también a la diversificar de proveedores y productos se suma la diversificación geográfica de mercados. Los autores Baldwin y Evenett (2020), toman como estrategia la diversificación geográfica de la producción.

Los resultados expuestos como recopilación de acciones adoptadas por empresas que participan en los mercados internacionales, permiten reforzar la idea de que la capacidad de adaptación a las transformaciones del ambiente de negocios, contribuye a la implementación de estrategias para el aprovechamiento de las oportunidades del mercado (Gallo, 2017; Carmona De Ríos, 2018). Además, según (Capa et al., 2018) "el entorno en el que se desenvuelven las empresas exportadoras, está caracterizado por una dinámica de cambios, donde los avances tecnológicos, la calidad, la innovación, la flexibilidad y el desempeño humano constituyen un reto permanente para mantener la competitividad" (p. 274). Más aún en una etapa de crisis sanitaria.

\section{Conclusión}

La crisis sanitaria asociada al COVID-19 ha representado enormes desafíos para las empresas exportadoras. Las medidas implementadas por los distintos gobiernos para frenar la expansión del virus, han implicado la interrupción de las cadenas de suministros. En forma paralela, los efectos económicos se han manifestado en la disminución de la demanda de productos a nivel internacional. No obstante, son notables los esfuerzos de las empresas exportadoras y de los gobiernos por no dejar caer sus ventas hacia el exterior.

Para Ecuador, China se constituye en el principal mercado para varios productos de exportación, pero los efectos de la pandemia han evidenciado la necesidad de diversificar los mercados. A la pregunta de investigación planteada, sobre el impacto de la crisis sanitaria en las exportaciones ecuatorianos, las estadísticas oficiales de los cuatro rubros analizados, permiten sostener que las empresas han podido mantener su operatividad para responder a la demanda de los mercados internacionales. Los productos banano y camarón siguen ocupando un papel prioritario en el ingreso de divisas, y pese a las restricciones propias de la pandemia, han registrado un incremento en el período de análisis. En el caso del rubro café y elaborado, se redujo en un $2 \%$ a diferencia del 2019. Lo que más se ha exportado es café procesado porque los productores no han dejado de abastecerse de la materia prima, es por ello que este sector se ha mantenido.

En cuanto al sector cacaotero se han registrado descensos en las cifras por el tema de la distribución de estos productos a pesar de que en los primeros meses se mostraba un crecimiento 
en comparación al 2019. El tonelaje de exportación fue mayor al del año anterior sin embargo se tenían proyecciones para esas fechas mucho más favorables que las presentadas.

Para cubrir el objetivo de investigación, se han identificado artículos científicos que retratan las decisiones empresariales para adaptarse a los cambios que ha supuesto la pandemia. En la exposición de los autores se destacan las estrategias de diversificación de mercados (Baldwin y Evenett, 2020), diversificación de proveedores para gestionar las interrupciones de la cadena de suministro (Sharma et al., 2020), certificaciones de cumplimiento de requisitos sanitarios y productos orgánicos (Barichello, 2020), la digitalización de actividades, fusionando el comercio y la tecnología (Oldekop et al., 2020).

Si bien estas estrategias no cubren todas las necesidades de las empresas exportadoras, pueden servir de referente para robustecer el aprendizaje en un escenario inesperado que ha afectado de forma desigual a las empresas alrededor del mundo. Frente a las nuevas formas de trabajar y a las transformaciones del ambiente de negocios, la capacidad de adaptación cobra mayor importancia para defender la permanencia en el mercado.

Las futuras líneas de investigación podrían enfocarse en analizar las experiencias de las empresas, mediante un enfoque cuantitativo que busque integrar la percepción de los exportadores sobre las dificultades afrontadas y adaptaciones organizacionales efectuadas para responder a las exigencias del entorno de negocios que ha representado el COVID-19.

\section{Referencias Bibliográficas}

Asociación Latinoamericana de Integración (2020). Tendencias del comercio. Editorial ALADI. http://www.aladi.org/sitioaladi/documentos/tenci/InformeTendenciasdelComercio.pdf

Alaña Castillo, T. P., Crespo García, M. K., y Gonzaga Añazco, S. J. (2018). ¿Cómo las estrategias empresariales permiten una ventaja competitiva en las micro, pequeñas y medianas empresas de la provincia de El Oro? Revista Universidad y Sociedad, 10(2), 257-262. http://scielo.sld.cu/scielo.php?script=sci_arttext\&pid=S2218-36202018000200257

Alvarado, P. (20 de Julio de 2020). Envío de camarón bajó 23\% en junio y en julio la caída se agudizará, según la Cámara Nacional de Acuacultura. Diario El Comercio. https://www.elcomercio.com/actualidad/envio-camaron-exportacion-caida-mercado.html

Amankwah-Amoah, J. (2020). Stepping Up and Stepping Out of COVID-19: New Challenges for Environmental Sustainability Policies in the Global Airline Industry. Journal of Cleaner Production, 271, 123000. https://doi.org/10.1016/j.jclepro.2020.123000

ANECACAO. (Septiembre de 2020). ANECACAO Asociación Nacional de Exportadores de cacao. http://www.anecacao.com/index.php/es/revista.html

Asociación de Exportadores de Banano del Ecuador. (2020). Enero-Mayo de 2020 Crecimiento del 10,33\% en exportaciones. Bananotas Bilingue, 142, 1-62. https://fb329f0a-8a6c-42169e2f-dcf8067bce4d.filesusr.com/ugd/f4cd67_cc3eaee12fe74a32b1848d35e74d2ba8.pdf

Asociación de Exportadores de Banano del Ecuador. (2020). Se detuvo el empuje inicial de este año. Bananotas Bilingue, 1-72. https://fb329f0a-8a6c-4216-9e2f- 
dcf8067bce4d.filesusr.com/ugd/f4cd67_37e633be2aa74b48a1f860bec100e3e4.pdf?index $=$ true

Baldwin, R., y Weder di Mauro, B. (2020). Economics in the Time of COVID-19. London. http://dln.jaipuria.ac.in:8080/jspui/bitstream/123456789/2757/1/Economics\%20in\%20the $\% 20$ Time $\% 20$ of\%20COVID-19.pdf

Baldwin, R., \& Evenett, S. (2020). COVID-19 and Trade Policy: Why Turning Inward Won't Work. London.

Banco Mundial. (2020). Global Economic Prospects. Washington, DC: Banco Mundial. https://openknowledge.worldbank.org/handle/10986/33748

Barichello, R. (2020). The COVID-19 pandemic: Anticipating its effects on Canada's agricultural trade. Canadian Journal Agricultural Economics, 2019-224. https://doi.org/10.1111/cjag.12244

Bonomelli, G. (2020). La pandemia del Coronavirus y su impacto sobre el comercio internacional: Una mirada sobre el mundo y Argentina. Revista Integración y Cooperación Internacional (30), $5-12$. http://rephip.unr.edu.ar/bitstream/handle/2133/18331/Art\%C3\%ADculo\%201\%20$\% 20$ Revista\%20Nro.\%2030.pdf?sequence=3

Capa Benítez, L. B., Benítez, R. M., y Capa Benítez, X. (2018). El liderazgo como fuente de ventaja competitiva para las organizaciones. Universidad y Sociedad, 10(2), 273-284. https://rus.ucf.edu.cu/index.php/rus/article/view/864

Carmona De Ríos, C. (2018). ¿Cuál es la importancia de implementar estrategias en las $\begin{array}{lllll}\text { organizaciones? Revista científica anfibios, } & \text { l(1), } & \text { 71-79. }\end{array}$ http://www.revistaanfibios.org/ojs/index.php/afb/article/view/20/16

Castro, C., Castañeda, Z., Ruiz, K. R., González, G., y Poveda, G. (2020). El sector florícola ecuatoriano y su afectación en el mercado internacional producto de la pandemia causada por el Covid-19. CONGRESO INTERNACIONAL VIRTUAL SOBRE COVID-19. CONSECUENCIAS PSICOLÓGICAS, SOCIALES, POLÍTICAS Y ECONÓMICAS, (págs. 53-65). Guayaquil. https://www.eumed.net/actas/20/covid/5-el-sector-floricolaecuatoriano-y-su-afectacion-en-el-mercado-internacional.pdf

CEPAL. (6 de Agosto de 2020). Comisión Económica para América Latina y el Caribe. (CEPAL, Ed.) Obtenido de Comisión Económica para América Latina y el Caribe: https://repositorio.cepal.org/bitstream/handle/11362/45877/S2000497_es.pdf?sequence=1 \&isAllowed $=\mathrm{y}$

CEPAL. (2020). Los efectos del COVID-19 en el comercio internacional y la logística. CEPAL. https://www.cepal.org/es/publicaciones/45877-efectos-covid-19-comercio-internacionalla-logistica

Chandler, A. (1962). Strategy and Estructure. Captar in the historia of the industrial Enterprise. Cambridge: The MIT Press. https://mitpress.mit.edu/books/strategy-and-structure

Edeh, J. O.-H. (2020). Efectos de las estrategias de innovación en el desempeño de las exportaciones: nueva evidencia empírica de empresas de mercados en desarrollo. Proyección tecnológica y cambio social, 158(120167). https://doi.org/10.1016 / j.techfore.2020.120167 
El Universo. (17 de julio de 2020). EL UNIVERSO. Obtenido de EL UNIVERSO: https://www.eluniverso.com/noticias/2020/07/17/nota/7908567/exportaciones-cafeecuador-reduccion-ingresos-sacos-pandemia

Foladori, G., y Delgado Wise, R. (2020). Para comprender el impacto disruptivo del covid-19, un análisis desde la crítica de la economía política. Revista Migración y Desarrollo, 18(34), 139-156.

Gallo Mendoza, J. G. (2017). La orientación al mercado, el dinamismo del entorno y la tipología estratégica de la pequeña y mediana industria: un análisis mediante modelo de ecuaciones $\begin{array}{lllll}\text { estructurales. INNOVA Research Journal, } & \text { 2(8), }\end{array}$ https://doi.org/10.33890/innova.v2.n8.1.2017.342

Gómez Rudy, C. M. (2020). Consideraciones para expandir su actividad comercial e impulsar su competitividad post COVID-19. Revista Científica CENTROS, IX(2), 158-177. Obtenido de https://revistas.up.ac.pa/index.php/centros

GRUSZCZYNSKI, L. (2020). The Covid-19 pandemic and international trade: Temporary turbulence or paradigm shift? European Journal of Risk Regulation, 337-342. doi:https://doi.org/10.1017/err.2020.29

Hannan, M. T., \& Freeman, J. (1977). The Population Ecology of Organizations. American Journal of Sociology, 82(5), 929-964.

He, H., \& Harris, L. (2020). The Impact of Covid-19 Pandemic on Corporate Social Responsibility and Marketing Philosophy. Journal of Business Research., 116, 176-182. https://doi.org/10.1016/j.jbusres.2020.05.030

Holguín, D. (2020). Con la red llena. Revista Vistazo, especial 500 mayores empresas del Ecuador, 204-206.

Kakoolaki, S., Ebne al-Torab, S., Ghajari, A., Anvar, A. A., Sepahdari, A., Ahari, H., \& Hoseinzadeh, H. (2020). Socio-economic impacts of Coronavirus (COVID-19) outbreak on world shrimp aquaculture sector. Iranian Journal of Aquatic Animal Health, 6(1), 1-18. http://ijaah.ir/article-1-212-en.html

Kerr, W. (2020). The COVID-19 pandemic and agriculture-Short and long run implications for international trade relations. Canadian Journal of Agricultural Economics/Revue canadienne d'agroeconomie., 225-229. https://doi.org/10.1111/cjag.12230

León Villamar, F., Calderón Salazar, J., y Mayorga Quinteros, E. (2016). Estrategias para el cultivo, comercialización y exportación del cacao fino de aroma en Ecuador. Ciencia UNEMI, 9(18), 45-55.

Loría Sagot, M. (2020). Crisis, reactivación económica y gestión pública por resultados ¿Cuáles son los desafíos? Logos, I(2), 13-23.

Love, D., Edward H, A., Asche, F., Belton, B., Cottrell, R., Froehlich, H., . . Zhang, W. (2020). SOC ARXIV. https://doi.org/0.31235/osf.io/x8aew

March, J., \& Simon, H. (1958). Organizations. New York: John Wiley \& Sons. https://www.worldcat.org/title/organizations/oclc/1329335

Martínez, C. N., y Rubio Bañón, A. (2020). Emprendimiento en épocas de crisis: Un análisis exploratorio de los efectos de la COVID-19. Small Business International Review, IV(2), 53-66. https://doi.org/10.26784/sbir.v4i2.279

Esta obra se comparte bajo la licencia Creative Common Atribución-No Comercial 4.0 International (CC BY-NC 4.0) 
Nicola, M., Alsafi, Z., Sohrabi, C., Kerwan, A., Al-Jabir, A., Iosifidis, C., . . Agha, R. (2020). The socio-economic implications of the coronavirus pandemic (COVID-19): A review. International journal of surgery, 78, 185-193. https://doi.org/10.1016 / j. ijsu.2020.04.018

Oldekop, J. A., Horner, R., Hulme, D., Adhikari, R., Agarwal, B., Alford, M., . . . Zhang, Y.-F. (2020). COVID-19 and the case for global development. Elsevier Ltd, 134. https://doi.org/10.1016/j.worlddev.2020.105044

Organización Mundial de Comercio. (22 de Junio de 2020). El comercio se contrae de forma acusada en la primera mitad de 2020. Organización Mundial de Comercio. https://www.wto.org/spanish/news_s/pres20_s/pr858_s.htm\#: :text=El\%20comercio\%20 mundial\%20se\%20contrajo,19\%20en\%201a\%20econom\%C3\%ADa\%20mundial.\&text= Con\%20arreglo\%20a\%20la\%20situaci\%C3\%B3n,se\%20cumpla\%201a\%20proyecci\%C3 $\%$ B3n\%20optimista.

Park, C.-Y., Kijin, K., Roth, S., Beck, S., Kang, J., Tayag, M., \& Griffin, M. (2020). Global Shortage of Personal Protective Equipment amid COVID-19: Supply Chains, Bottlenecks, and Policy Implications. ASIAN DEVELOPMENT BANK, 1-10. http://dx.doi.org/10.22617/BRF200128-2

Pereira, E., Loureiro, I., Ribeiro, P., Costa, S., Costa, N., \& Arezes, P. (28 de Febrero de 2019). Sustainable Business Strategies: What You Think Is What You Do?. Occupational and Environmental Safety and Health, 747-755. https://doi.org/10.1007/978-3-030-147303_78

Pesantez Calva, A. E., Romero Correa, J. A., y González-Illescas, M. L. (2020). Comercio electrónico B2B como estrategia competitiva en el comercio internacional: Desafíos para $\begin{array}{llll}\text { Ecuador. INNOVA } & \text { Research }\end{array}$ https://doi.org/10.33890/innova.v5.n1.2020.1166

Quevedo Barros, M. R., Vásquez Lafebre, L. M., Quevedo Vázquez, J. O., y Pinzón Prado, L. T. (2020). COVID-19 y sus efectos en el comercio internacional. Caso Ecuador. Dominio de las Ciencias, VI(3), 1006-1015. https://dx.doi.org/10.23857/dc.v6i3.1330

Saavedra Calderón, J. A. (2020). El covid-19 en américa latina: situación y desafíos del sistema internacional. Cuadernos de Derecho Público, 51-59. https://doi.org/10.22529/cdp

Sharma, A., Adhikary, A., \& BikashBorah, S. (2020). Covid-19's impact on supply chain decisions: Strategic insights from NASDAQ100 firms using Twitter data. Journal of Business Research ELSEVIER, 443-449. https://doi.org/10.1016/j.jbusres.2020.05.035

Slusarczyk Antosz, M., y Morales Merchán, N. H. (2016). Análisis de las estrategias empresariales y de las TIC. 3C Empresa. Investigación $Y$ Pensamiento Crítico, 5(1), 29-46. https://doi.org/10.17993/3cemp.2016.050125.29-46

Sorroza Rojas, N. A., Jinez Jinez, H. E., Jinez Sorroza, L. D., y Jinez Sorroza, B. E. (2020). Impacto de las pandemias en el comercio internacional y Ecuador. RECIAMUC, IV(2), 1220. https://doi.org/10.26820/reciamuc/4.(2).abril.2020.12-20

Subdirección General de Estudios y Evaluación de Instrumentos de Política Comercial. (2020). EL COMERCIO EXTERIOR ANTE LOS RETOS DE 2020. BOLETÍN ECONÓMICO DE ICE 3121, 3-17. https://doi.org/10.32796/bice.2020.3121.6983

Verdugo Morales, N., y Andrade Díaz, V. (2018). Productos tradicionales y no tradicionales del Ecuador: Posicionamiento y eficiencia en el mercado internacional para el período 2013 - 
2017. X-Pedientes Económicos, II(3), 84-102. http://ojs.supercias.gob.ec/index.php/Xpedientes_Economicos/article/view/43/16

Zelicovich, J., Romero, C., y Busso, A. (2020). El impacto del COVID-19 en las relaciones comerciales internacionales. Centro de Investigaciones en Política y Economía Internacional, https://ri.conicet.gov.ar/bitstream/handle/11336/111795/CONICET_Digital_Nro.135b6b5 c-329a-485b-829f-962e1b13b9e3_A-44-52.pdf?sequence=5\&isAllowed=y 